[19] M. Lemoigne, A. Sanchez et H. Girard. C. R. Ac. Agric., 1952, 38, 606 .

[20] G. Mocquot, R. Chevalier, R. Barnier, J. Auclatr et C. Alais. C. R. Ac. Agric., 1949, 35, 30.

[21] W. Монr et K. Konnen. Die Molkerei Zeit., 1950, 71, 1035.

[22] P. Morel. Thèse de Doctorat Vétérinaire, Lyon, 1962.

[23] - Le Lait, 1962, 42, 593.

[24] C. Neal et M. Calbert. J, Dairy Sc, 1955, 38, 629.

[25] J. Pien, J. Lignac et P. Claude. Ann. Falsif. et Fraudes, 1953, 46, 258.

[26] A. Sanchez et P. Lamensans. Ann. Inst. Pasteur, 1948, 74, 142.

[27] G. Silverman et F. Kosikowsky. J. Milk and Food Techn., 1952, 15,20 .

\title{
LA POLLUTION RADIOACTIVE DU LAIT CONSOMMÉ DANS L'AGGLOMÉRATION PARISIENNE
}

par

\author{
C. Madelmont et G. Michon
}

Les explosions aériennes des armes nucléaires libèrent dans la stratosphère et dans l'atmosphère des produits de fission qui donnent lieu, à plus ou moins longue échéance, à des retombées radioactives terrestres. Celles-ci contaminent le sol, les plantes, les animaux et l'homme par l'intermédiaire de la chaîne alimentaire.

Cette contamination se retrouve en particulier dans le lait. Principal vecteur alimentaire $d u$ calcium dans l'alimentation européenne, il est important pour des questions d'ordre sanitaire d'en connaître sa teneur en ${ }^{90} \mathrm{Sr}$ et ${ }^{137} \mathrm{Cs}$ pour les éléments à vie longue et en ${ }^{131} \mathrm{I}$ et ${ }^{89} \mathrm{Sr}$ pour les éléments à vie courte et moyenne.

Nous avons cherché à obtenir quelques informations sur la teneur en ces divers radionuclides du lait distribué dans l'agglomération parisienne. La présente étude porte sur les années 1961 et 1962 .

\section{Prélèvements.}

Les prélèvements ont été effectués par les soins du Laboratoire du lait du Service Vétérinaire Sanitaire de Paris et du département de la Seine (1) à la faveur d'autres prélèvements en vue de l'ins-

(1) Nous remercions très vivement $M M$. Basille et Hautefort qui ont assuré les prélèvements de lait, ainsi que Mme Augustin et $M$. Artaud qui ont prêté leur concours technique à la réalisation des dosages. 
pection sanitaire. Tous les quinze jours, un échantillon de 20 litres de lait de grand mélange représentant soit un seul département, soit le mélange de lait de deux ou trois départements a été ainsi obtenu suivant les possibilités et les besoins du service préleveur.

\section{Méthode d'analyse.}

La recherche $d u{ }^{90} \mathrm{Sr}$ et $d u{ }^{89} \mathrm{Sr}$ est effectuée selon la technique de JeANMAIRE et Michon [1] qui est basée sur la séparation nitrique $\mathrm{du}$ strontium entraîneur suivie d'une purification sur résine échangeuse d'ions.

La détermination sur des installations de mesure à bas mouvement propre de $1^{\prime 90} \mathrm{Y}$ en équilibre radioactif avec le ${ }^{90} \mathrm{Sr}$ permet de remonter à l'activité du ${ }^{90} \mathrm{Sr}$ et par différence au ${ }^{89} \mathrm{Sr}$.

La détermination $d u{ }^{137} \mathrm{Cs}$ et de $\mathrm{l}^{131} \mathrm{I}$ est faite par spectrométrie $\gamma$ après étalonnage adéquat du sélecteur d'amplitude [2].

\section{Représentativité de l'échantillonnage.}

D'avril 1961 à décembre 1962, 45 échantillons ont été analysés. Ils représentaient les divers départements suivants :

$\begin{array}{lrlll}\text { Oise } \ldots \ldots \ldots & 19 \text { fois } & \text { Eure-et-Loir . } & 8 \text { fois } \\ \text { Seine-et-Marne } & 8 \text { fois } & \text { Sarthe ..... } & 2 \text { fois } \\ \text { Eure } \ldots \ldots \ldots & 12 \text { fois } & \text { Loir-et-Cher . } & 4 \text { fois } \\ \text { Seine-et-Oise } & 2 \text { fois } & \text { Loiret ...... } & 5 \text { fois }\end{array}$

Ces départements forment une ceinture autour du département de la Seine et couvrent le Bassin Parisien, zone relativement homogène au point de vue pédoclimatique. Sur le plan de la production laitière destinée en nature à la consommation humaine, d'après le Bulletin Municipal Officiel de la ville de Paris, les départements échantillonnés comportent 72 ateliers de pasteurisation sur un total de 137 qui alimentent plus ou moins régulièrement la région parisienne, soit 52 p. 100.

Cependant le sondage effectué suivant les disponibilités du laboratoire a conduit à une certaine disparité dans les fréquences des prélèvements rapportés à un département, ce qui complique l'interprétation des résultats. Il serait préférable de disposer d'un échantillonnage pondéré par rapport aux diverses contributions de ces départements à l'approvisionnement de Paris. 


\section{Résultats.}

Le tableau no 1 fournit l'ensemble des valeurs obtenues pour le ${ }^{90} \mathrm{Sr}$, le ${ }^{137} \mathrm{Cs}$, le ${ }^{89} \mathrm{Sr}$ et l'131 I, échantillon par échantillon.

\section{a) Iode 131 .}

Le type de sondage adopté pour la région parisienne n'est pas adapté à une étude sérieuse de la pollution du lait par les radioéléments de courte période [3]. Cependant quelques résultats figurent dans le tableau et permettent de dégager quelques données générales. Les périodes où l'iode est présent dans le lait en quantité notable correspondent aux semaines qui suivent les séries d'explosions aériennes soviétiques : de septembre à octobre 1961, de août à novembre 1962 .

Les valeurs obtenues peuvent être comparées avec celles obtenues lors d'un contrôle systématique de la pollution du lait par l'iode [4 et 5]. C'est ainsi que le lait prélevé aux Halles le 28 octobre 1961 présente une teneur de 260 pCi d'131I par litre, et un lait prélevé en Eure-et-Loir le 30 octobre 1961 présente une teneur de 280 pCi/litre.

La série de tests aériens américains dans l'hémisphère sud n'a pas entraîné de pollution décelable par l'iode 131.

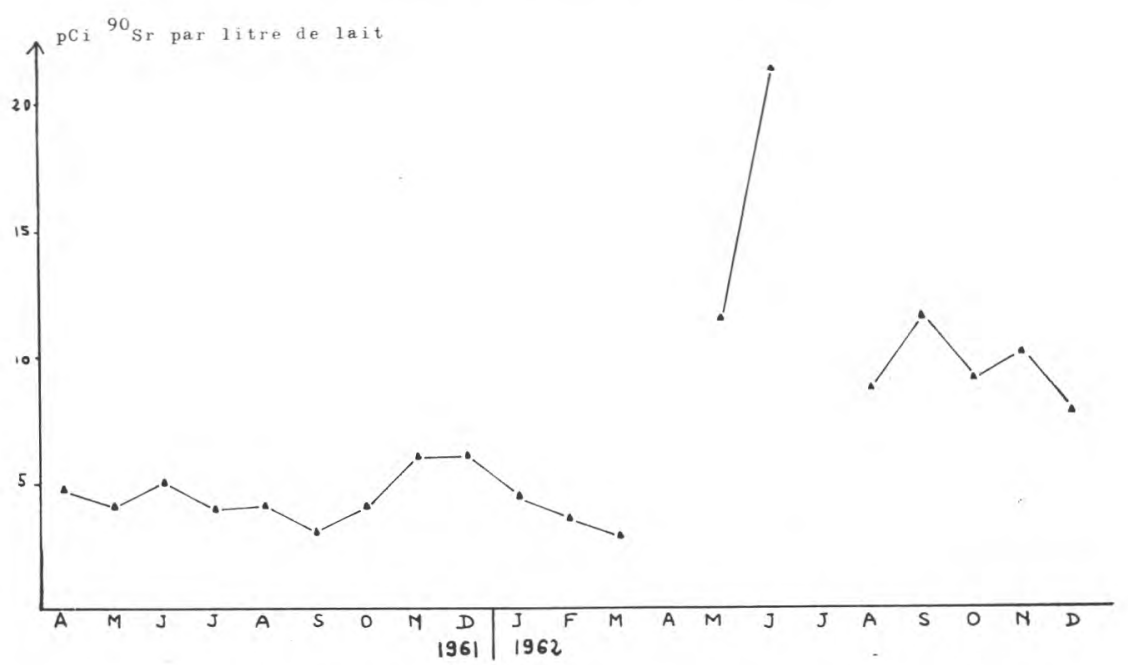

Fig. 1. - Moyenne de la contamination par le ${ }^{90} \mathrm{Sr}$ observée sur les échantillons analysés. 


\section{b) Strontium 90 et Césium 137.}

Ces deux radioéléments ont des périodes longues et leurs teneurs dans le lait ne varient pas de façon brusque. Des prélèvements espacés dans le temps peuvent refléter l'évolution de la pollution du lait par ces nuclides. Les figures 1 et 2 montrent comment ont évolué les teneurs moyennes pour le lait consommé à Paris. L'allure générale des courbes est assez semblable à celles obtenues en mesurant les teneurs en ${ }^{90} \mathrm{Sr}$ et ${ }^{137} \mathrm{Cs}$ de l'air et de l'eau de pluie [6]. La reprise des essais nucléaires en septembre 1961, qui se manifeste par une recrudescence des retombées radioactives surtout due à l'apparition de corps à vie courte, n'a qu'une faible action sur les teneurs en ${ }^{90} \mathrm{Sr}$ et ${ }^{137} \mathrm{Cs} \mathrm{du}$ fait de la petite contribution de ces corps à la radioactivité globale des retombées dans les mois qui suivent la série de tests. De plus, à cette époque, bon nombre de troupeaux sont à l'étable, nourris avec des aliments récoltés antérieurement à la période des retombées et par conséquent peu pollués.

Par contre, une augmentation très nette se manifeste au printemps de l'année 1962. Cette augmentation est due, d'une part aux retombées radioactives stratosphériques consécutives aux explosions de l'automne et, d'autre part au fait que les troupeaux sont alors nourris avec des fourrages verts exposés à ces retombées.

L'influence de la série de tests américains dans l'océan Pacifique en mai 1962, si elle existe, ne peut être dissociée du phénomène précédent. Enfin, la série de tests soviétiques de août à novembre 1962 n'entraîne pas de variation manifeste de la teneur en ${ }^{90} \mathrm{Sr}$

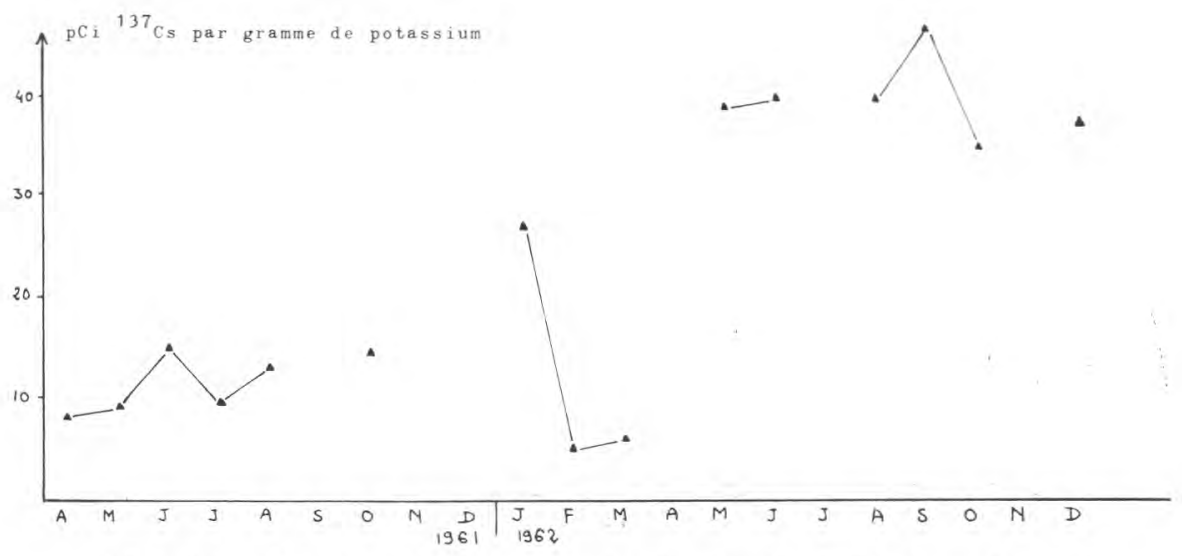

Fig. 2. - Moyenne de la contamination par le ${ }^{137} \mathrm{Cs}$ observée sur les échantillons analysés. 
et en ${ }^{137} \mathrm{Cs}$. Il n'en ira probablement pas de même au printemps de l'année 1963.

Certains résultats concernant le césium manquent pour la fin de l'année 1961. A cette époque nous utilisions pour déshydrater le lait un appareil nécessitant de grande quantité d'air. Cet air étant pollué par les retombées, nous avons ainsi provoqué une contamination supplémentaire du lait et nous avons préféré ne pas tenir compte de résultats très aléatoires du fait de la présence de radioéléments gênants pour la mesure spectrométrique du césium.

L'examen du tableau montre une bonne uniformité des résultats d'un département à un autre. Ceci tient sans doute à l'unité climatique et géologique du Bassin Parisien et nous a autorisé à calculer malgré l'hétérogénéité de l'échantillonnage, des moyennes pour l'ensemble de la consommation parisienne.

\section{c) Strontium 89}

En ce qui concerne le strontium 89, corps à vie moyenne (55 jours) la situation est intermédiaire entre le cas de l'iode 131 et celui du strontium 90. Ayant complètement disparu après les explosions de 1958, il réapparaît dans les retombées troposphériques consécutives aux explosions de septembre 1961, apparaît dans le lait dès octobre de la même année, diminue au cours de l'hiver $d u$ fait $d u$ changement d'alimentation, pour réapparaître dans le lait quand les animaux retournent aux pâturages. Pendant toute cette période, la radioactivité due au ${ }^{89} \mathrm{Sr}$ a décru avec la période de 55 jours et il en subsiste cependant encore au moment de la série d'août 1962 .

\section{Interprétations sanitaires.}

Le tableau no 2 résume les moyennes que nous avons pu calculer pour la région parisienne.

En ce qui concerne l'iode 131, nous n'avons pas calculé de moyenne. Les valeurs observées sont de l'ordre de grandeur de celles observées par ailleurs (4-5) et l'on peut conclure que la pollution par l'iode n'a pas donné lieu, pour l'agglomération parisienne et pour la période envisagée, à une situation critique du point de vue sanitaire.

En ce qui concerne le strontium 89 et 90 , bien qu'il n'existe pas de quantité maximale admissible définie pour le lait, on peut 
TABLEAU I

\begin{tabular}{|c|c|c|c|c|c|}
\hline Mois & $\begin{array}{l}{ }^{90} \mathrm{Sr}(2) \\
\mathrm{pCi}(1) / 1 \\
\text { de lait }\end{array}$ & $\begin{array}{l}{ }^{89} \mathrm{Sr}(2) \\
\mathrm{pCi} / \mathrm{l} \text { de } \\
\text { lait }\end{array}$ & $\begin{array}{c}{ }^{137} \mathrm{Cs} \\
\mathrm{pCi} / \mathrm{g} \text { de } \\
\text { potassium }\end{array}$ & $\begin{array}{l}{ }^{131} \mathrm{I} \\
\mathrm{pCi} / \mathrm{l} \text { de } \\
\text { lait }\end{array}$ & Origine \\
\hline 1961 & & & & & \\
\hline Avril $\ldots$ & $\begin{array}{l}5,0 \\
5,6 \\
4,9 \\
3,8\end{array}$ & & $\begin{array}{r}7 \\
11 \\
8 \\
6\end{array}$ & & $\begin{array}{l}\text { Oise } \\
\text { Eure } \\
\text { Seine-et-Marne et Loiret } \\
\text { Eure }\end{array}$ \\
\hline Mai ..... & $\begin{array}{l}4,9 \\
3,3\end{array}$ & & $\begin{array}{l}9 \\
9\end{array}$ & & $\begin{array}{l}\text { Oise } \\
\text { Oise }\end{array}$ \\
\hline Juin .... & $\begin{array}{l}4,6 \\
3,7 \\
7,1\end{array}$ & & $\begin{array}{c}13 \\
\text { mesure non } \\
\text { effectuée } \\
17\end{array}$ & & $\begin{array}{l}\text { Oise } \\
\text { Seine-et-Marne } \\
\text { Eure-et-Loir }\end{array}$ \\
\hline Juillet . . & $\begin{array}{l}4,3 \\
3,8 \\
4,5 \\
2,5\end{array}$ & & $\begin{array}{l}13 \\
11 \\
11 \\
8\end{array}$ & & $\begin{array}{l}\text { Oise } \\
\text { Eure } \\
\text { Seine-et-Marne, Loir-et-Cher et } \\
\quad \text { Loiret } \\
\text { Oise }\end{array}$ \\
\hline Août $\ldots$. & $\begin{array}{l}3,3 \\
4,5\end{array}$ & & $\begin{array}{l}11 \\
14\end{array}$ & & $\begin{array}{l}\text { Oise } \\
\text { Eure-et-Loir, Sarthe et Loiret }\end{array}$ \\
\hline Septembre. & $\begin{array}{l}3,6 \\
2,8 \\
3,0\end{array}$ & $\begin{array}{l}6 \\
3\end{array}$ & $\begin{array}{c}\text { contamination } \\
" \\
"\end{array}$ & $\begin{array}{r}85 \\
312 \\
62\end{array}$ & $\begin{array}{l}\text { Oise } \\
\text { Eure } \\
\text { Seine-et-Marne }\end{array}$ \\
\hline Octobre .. & $\begin{array}{l}3,1 \\
4,0 \\
3,9 \\
6,3\end{array}$ & $\begin{array}{r}29 \\
18 \\
31 \\
119\end{array}$ & $\begin{array}{c}\text { contamination } \\
14,5 \\
\text { contamination } \\
"\end{array}$ & $\begin{array}{l}119 \\
198 \\
227 \\
260\end{array}$ & $\begin{array}{l}\text { Oise } \\
\text { Eure } \\
\text { Seine-et-Marne, Loir-et-Cher et } \\
\quad \text { Loiret. } \\
\text { Eure-et-Loir }\end{array}$ \\
\hline Novembre & $\begin{array}{l}6,9 \\
5,3\end{array}$ & $\begin{array}{l}98 \\
95\end{array}$ & contamination & $\begin{array}{l}182 \\
299\end{array}$ & $\begin{array}{l}\text { Oise } \\
\text { Eure }\end{array}$ \\
\hline Décembre. & $\begin{array}{l}8,2 \\
4,0\end{array}$ & $\begin{array}{l}141 \\
181\end{array}$ & contamination & $\begin{array}{r}135 \\
0\end{array}$ & $\begin{array}{l}\text { Eure-et-Loir } \\
\text { Eure-et-Loir }\end{array}$ \\
\hline
\end{tabular}


TABLEAU I (suite)

\begin{tabular}{|c|c|c|c|c|c|}
\hline Mois & $\begin{array}{l}{ }^{90} \mathrm{Sr}(2) \\
\mathrm{pCi}(1) / 1 \\
\text { de lait }\end{array}$ & $\begin{array}{l}{ }^{89} \mathrm{Sr}(2) \\
\mathrm{pCi} / 1 \mathrm{de} \\
\text { lait }\end{array}$ & $\begin{array}{c}{ }^{137} \mathrm{Cs} \\
\mathrm{pCi} / \mathrm{g} \text { de } \\
\text { potassium }\end{array}$ & $\begin{array}{l}{ }^{131} \mathrm{I} \\
\mathrm{pCi} / \mathrm{l} \text { de } \\
\text { lait }\end{array}$ & Origine \\
\hline 1962 & & & & & \\
\hline Janvier ... & $\begin{array}{l}4,8 \\
3,9 \\
5,5 \\
3,9\end{array}$ & $\begin{array}{r}15 \\
10 \\
11 \\
0\end{array}$ & $\begin{array}{l}28 \\
47 \\
24 \\
12\end{array}$ & $\begin{array}{r}71 \\
0 \\
0 \\
0\end{array}$ & $\begin{array}{l}\text { Oise } \\
\text { Seine-et-Marne } \\
\text { Eure-et-Loir } \\
\text { Oise }\end{array}$ \\
\hline Février $\ldots$ & $\begin{array}{l}6,0 \\
2,8 \\
3,8\end{array}$ & & $\begin{array}{c}\text { contamination } \\
5\end{array}$ & & $\begin{array}{l}\text { Eure } \\
\text { Seine-et-Marne, Loir-et-Cher et } \\
\quad \text { Loiret }\end{array}$ \\
\hline Mars ..... & 2,9 & & 6 & & Oise \\
\hline Mai ..... & $\begin{array}{r}8,4 \\
12,6\end{array}$ & $\begin{array}{l}43 \\
69\end{array}$ & $\begin{array}{l}36 \\
40\end{array}$ & & $\begin{array}{l}\text { Oise } \\
\text { Eure-et-Loir, Loir-et-Cher et Loi- } \\
\quad \text { ret }\end{array}$ \\
\hline Juin $\ldots$. & 21,4 & 72 & 40 & & Oise et Eure \\
\hline Août $\ldots$. & 8,7 & 36 & 40 & & Oise et Seine-et-Marne \\
\hline Septembre & $\begin{array}{r}8,0 \\
15,4\end{array}$ & $\begin{array}{l}25 \\
92\end{array}$ & $\begin{array}{l}41 \\
54\end{array}$ & 120 & $\begin{array}{l}\text { Oise } \\
\text { Eure }\end{array}$ \\
\hline Octobre .. & $\begin{array}{l}9,0 \\
8,9 \\
9,7\end{array}$ & $\begin{array}{l}57 \\
61 \\
33\end{array}$ & $\begin{array}{l}37 \\
32\end{array}$ & & $\begin{array}{l}\text { Eure } \\
\text { Eure et Oise } \\
\text { Seine-et-Oise }\end{array}$ \\
\hline Novembre & 10,3 & 43 & & 39 & Seine-et-Oise \\
\hline Décembre. & 7,8 & 26 & 38 & & Eure \\
\hline
\end{tabular}

(1) $\mathrm{pCi}=10^{-12}$ Curie.

(2) Moyenne de deux analyses. 
TABLEAU II

\begin{tabular}{|c|c|c|c|}
\hline Mois & $\begin{array}{c}{ }^{90} \mathrm{Sr} \\
\mathrm{pCi} / \text { litre }\end{array}$ & $\begin{array}{c}{ }^{89} \mathrm{Sr} \\
\text { pCi/litre }\end{array}$ & $\begin{array}{c}{ }^{137} \mathrm{Cs} \\
\text { pCi/litre }\end{array}$ \\
\hline 1961 & & & \\
\hline Avril $\ldots \ldots \ldots$ & 4,8 & - & 8 \\
\hline Mai $\ldots . . . \ldots$ & 4,1 & - & 9 \\
\hline Juin $\ldots \ldots \ldots$ & 5,1 & - & 15 \\
\hline Juillet . . . . . & 4,0 & - & 9,6 \\
\hline Août $\ldots \ldots \ldots$ & 4,2 & - & 13,2 \\
\hline Septembre .... & 3,1 & 3 & contamination \\
\hline Octobre ...... & 4,1 & 43 & 14,5 \\
\hline Novembre..... & 6,1 & 96 & contamination \\
\hline Décembre ..... & 6,1 & 161 & contamination \\
\hline 1962 & & & \\
\hline Janvier $\ldots \ldots$ & 4,5 & 9 & 27 \\
\hline Février $\ldots \ldots \ldots$ & 3,6 & - & 5 \\
\hline Mars $\ldots \ldots \ldots$ & 2,9 & - & 6 \\
\hline Avril $\ldots \ldots \ldots$ & \multicolumn{2}{|c|}{ pas d'échantillons } & \\
\hline Mai $\ldots \ldots \ldots$ & 11,5 & 62 & 39 \\
\hline Juin $\ldots \ldots \ldots$ & 21,4 & 72 & 40 \\
\hline Juillet . . . . . & \multicolumn{2}{|c|}{ pas d'échantillons } & \\
\hline Août $\ldots \ldots \ldots$ & 8,7 & 36 & 40 \\
\hline Septembre .... & 11,7 & 58 & 47 \\
\hline Octobre ...... & 9,1 & 53 & 35 \\
\hline Novembre ..... & 10,3 & 43 & \\
\hline Décembre ..... & 7,8 & 26 & 38 \\
\hline
\end{tabular}

admettre en première approximation que si la teneur rapportée au gramme de calcium alimentaire ne dépasse pas :

pour le ${ }^{90} \mathrm{Sr} \quad 800 \mathrm{pCi} / \mathrm{g}$ de $\mathrm{Ca}$, pour le ${ }^{89} \mathrm{Sr} 1600 \mathrm{pCi} / \mathrm{g}$ de $\mathrm{Ca}$,

en moyenne sur toute la durée de la vie, on ne dépassera à aucun moment la charge corporelle entraînant une irradiation égale à l'irradiation maximale admissible.

Les valeurs figurant au tableau $n^{\circ} 2$ sont rapportées au litre de lait. Rapportées au gramme de $\mathrm{Ca}$, elles seront plus petites et leur comparaison avec les valeurs précédemment évoquées permettent d'apprécier le risque sanitaire encouru par la consommation du lait dans la région parisienne. 
En ce qui concerne le ${ }^{137} \mathrm{Cs}$, la seule référence que nous puissions évoquer est la concentration maximale admissible dans l'eau de boisson. Celle-ci a été fixée à $2 \cdot 10^{-5} \mu \mathrm{Ci} / \mathrm{cm}^{3}$ [7], soit $20000 \mathrm{pCi}$ par litre. Les teneurs observées dans le lait sont très loin de ces valeurs.

\section{$*^{*} *$}

Bien que l'échantillonnage n'ait pas été mené de façon très rationnelle, l'ensemble des résultats obtenus, en bon accord avec d'autres données concernant la pollution de l'air et de l'eau de pluie dans la région parisienne, avec les teneurs observées dans le lait d'autres régions de la France, permet de suivre les fluctuations de la pollution radioactive $\mathrm{du}$ lait et de faire des estimations moyennes sur le plan sanitaire.

Pendant la période d'avril 1961 à décembre 1962, les teneurs en radioéléments du lait sont restées dans des limites radiologiquement acceptables.

\section{BIBLIOGRAPHIE}

[1] L. Jeanmaire et G. Michon. Recherche et dosage des isotopes radioactifs 89 et 90 du Strontium dans le lait en poudre. Le Lait, juilletaoût $1959,39,369$ à 381 .

[2] L. Jeanmaire, H. Fourniguet et G. Michon. Dosage du ${ }^{137} \mathrm{Cs}$ dans le lait en poudre. Le Lait, novembre-décembre 1959, 39, 595 à 600 .

[3] G. Michon. Organisation d'un contrôle de la pollution radioactive du lait. Cas des radioisotopes de courtes périodes. Bull. Acad. Vét. France, 1963, 36, 6, 283-285.

[4] L. Jeanmaire et G. Michon. Les pollutions radioactives par l'iode 131

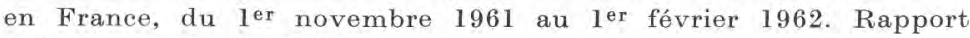
C.E.A., n ${ }^{\circ} 2223.1962$.

[5] M. L. Audren et Coll. Contrôle de la pollution radioactive du lait par $1^{131}$ I. Bull. Acad. Vét. France, 1963, 36, 6, 287-290.

[6] J.-C. Рнгцгрот. Bulletin d'Informations Scientifiques et Techniques $1963,75,135-156$.

[7] Protection contre les rayonnements. Recommandations de la Commission internationale de protection radiologique. Rapport du Comité II sur la dose admissible en cas d'irradiation interne, publication CIPR 2, 1959. Paris, Gauthier-Villard, 1963. 